\title{
OPTICS AND DYNAMIC APERTURE STUDIES FOR SYNCHROTRON RADIATION SOURCE DELSY
}

\author{
P.Beloshitsky, I.Meshkov, I.Titkova, JINR, Dubna, Russia
}

\section{Abstract}

The project DELSY, a $1.2 \mathrm{GeV}$ synchrotron radiation source [1] is being under development at JINR, Dubna, Russia. This source is dedicated to the investigations on condensed matter physics, atomic physics, biology, medicine, chemistry, micromechanics, lithography and others. For DELSY storage ring the layout with four straight sections was chosen. Every quadrant consists of MBA-structure and two halves of straight sections. The circumference of the ring is about $136 \mathrm{~m}$; the emittance of the electron beam is $11.4 \mathrm{~nm}$. The effects of the very strong wiggler of $10 \mathrm{~T}$ and undulator $(0.75 \mathrm{~T}, 150$ periods $)$ on beam parameters are discussed. The results of influence of machine imperfections on the optics properties are given.

\section{INTRODUCTION}

Project DELSY is based on the accelerator facility presented to JINR by the NIKHEF (Amsterdam). The NIKHEF accelerator facility consists of the linear electron accelerator MEA with the energy of electrons of $700 \mathrm{MeV}$ and the electron storage ring AmPS with the maximum energy of $900 \mathrm{MeV}$ and the beam current of $200 \mathrm{~mA}$.

The DELSY lattice is prepared in a way to use most of the AmPS ring magnetic elements but with significantly modified optics.

The construction of the DELSY facility will be proceeded in three phases:

- Phase I will be accomplished with the construction of a complex of free electron lasers covering continuously the spectrum from far infrared down to ultraviolet (of about $150 \mathrm{~nm}$ ).

- Phase II will be accomplished with the commissioning of the storage ring DELSY.

- Complete commissioning of the DELSY project will take place after finishing Phase III construction of an X-ray free electron laser. This phase is considered as the ultimate goal of the project.

\section{STORAGE RING}

\subsection{Lattice and basic parameters}

In comparison with [2], the lattice is simplified by excluding one quadrupoles family. The periodicity of the ring is 2 now. Every quadrant consists of the MBA structure: two halves of straight sections and two periodic cells. The periodic cell consists of two dipoles and three quadrupoles. The matching cell contains two dipoles and provides zero dispersion in the straight section. The particular values of the beta functions in the straight sections are adjusted by using doublet.

The main machine parameters are given in Table 1.

Table 1: Main parameters of the DELSY ring.

\begin{tabular}{|l|c|}
\hline Full energy, GeV & 1.2 \\
\hline Injection energy, GeV & 0.8 \\
\hline Circumference, $\mathrm{m}$ & 136.04 \\
\hline Bending radius, $\mathrm{m}$ & 3.3 \\
\hline Betatron tunes, $\mathrm{h} / \mathrm{v}$ & $9.44 / 3.42$ \\
\hline Momentum compaction factor & $5.03 \cdot 10^{-3}$ \\
\hline Natural chromaticity, $\mathrm{h} / \mathrm{v}$ & $-22.2 /-12.6$ \\
\hline Injection current, $\mathrm{mA}$ & 10 \\
\hline Stored electron current, $\mathrm{mA}$ & 300 \\
\hline Horizontal emittance, $\mathrm{nm}$ & 11.4 \\
\hline RF frequency, $\mathrm{MHz}$ & 476 \\
\hline Harmonic number & 216 \\
\hline Energy loss per turn, $\mathrm{keV}$ & 55.7 \\
\hline
\end{tabular}

The beta functions in a very strong wiggler must be small enough to avoid emittance increase and to minimise the optics distortions with the wiggler on. In our case $\beta_{x}=1.05 \mathrm{~m}$ and $\beta_{y}=2.80 \mathrm{~m}$ (Fig. 1 ).

The vertical beta function in the centre of the undulator must be small to provide the tolerable lifetime limited by the residual gas scattering. It was accepted to be $\beta_{x}=14.55$ $m$ and $\beta_{y}=0.98 m$ (Fig.2).

In another "undulator" quadrant the injection septum is placed. Two injection kickers separated in $9 \pi$ betatron phase advance are located in "wiggler" quadrants.

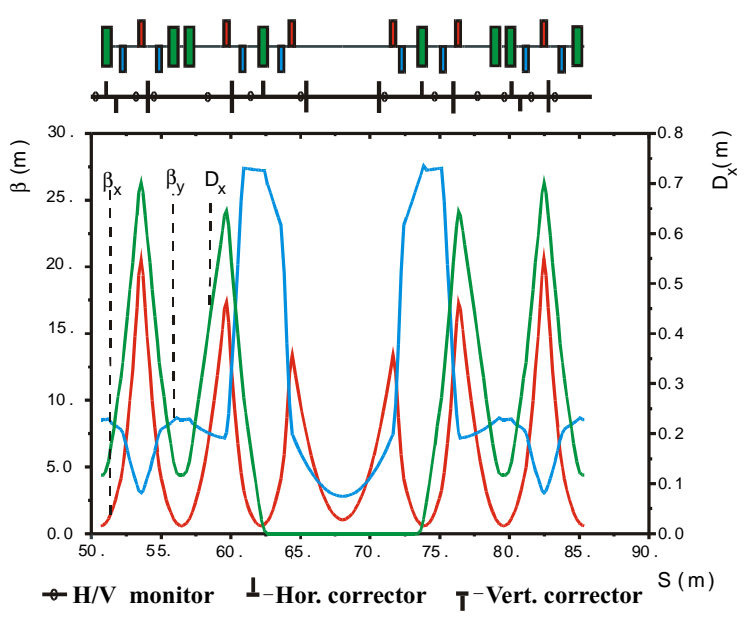

Figure 1: Lattice functions in the "wiggler" quadrant. 


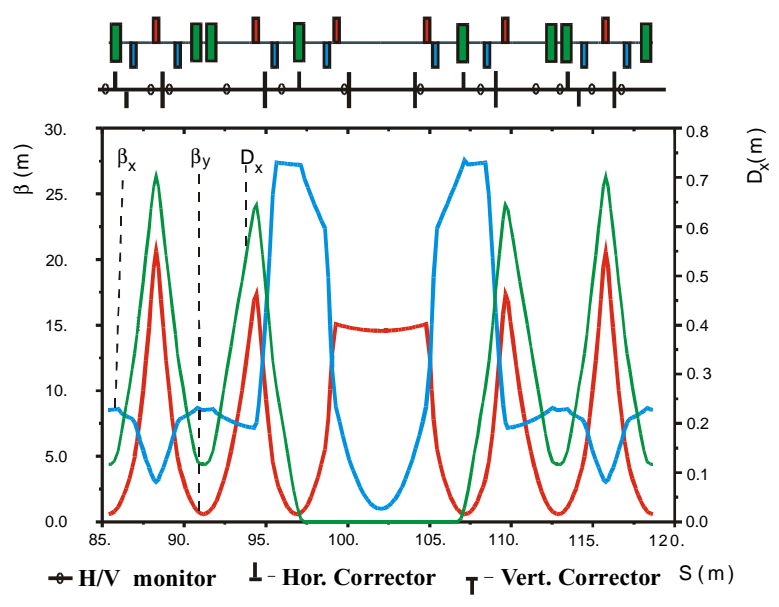

Figure 2: Lattice functions in the "undulator" quadrant.

\subsection{The influence of the insertion devices on the linear optics}

The measured multipole components of the $10 \mathrm{~T}$ wiggler [3] were used for the linear optics and dynamic aperture calculations with the wiggler on. The very strong wiggler produces great distortions of the linear optics. To minimise them, the following procedure was used. First, the strengths of two quadrupoles in the doublet matching the wiggler section were modified to maintain constraints $\left(\alpha_{x}=\alpha_{y}=0\right)$ with the wiggler on and off. This prevents the beating of the beta functions everywhere outside the wiggler section. After this the machine tunes are changed significantly. To bring them back as well as to maintain the required beta functions in the straight sections, a global matching procedure involving all matching doublets and two quadrupoles of the matching cells was used. As a result, the deviation of the beta functions for the machine with the wiggler on from that one with the wiggler off is less than $7 \%$, the emittance is increased up to $21.3 \mathrm{~nm}$ (Fig.3).

The same procedure was carried out for the undulator $(0.75 \mathrm{~T}, 150$ periods of $2.25 \mathrm{~cm})$, but its effect on the machine optics is much smaller. The deviation of the beta functions for the machine with the undulator on is less than $1 \%$; the emittance is decreased down to $11.14 \mathrm{~nm}$.

\subsection{Dynamic aperture}

The injection energy for DELSY is $0.8 \mathrm{GeV}$, while operation is at $1.2 \mathrm{GeV}$. This imposes strong constraints on the DELSY dynamic aperture. The solution with two sextupole families was found that solves the problem. First, phase advances of the periodic cell were chosen to provide a small emittance at moderate natural chromaticities. Then the positions of the sextupoles in the matching cells were adjusted. As a result, the dynamic aperture that calculated with the MAD computer code [4] is $99 \sigma_{x}$ and $87 \sigma_{y}$ respectively (without influence of the insertion devices). For the particles with the momentum deviation $\Delta \mathrm{p}=+1 \%$ the dynamic aperture decreases to $71 \sigma_{x}$ and $81 \sigma_{y}$, for the particles with $\Delta \mathrm{p}=-1 \%$ to $70 \sigma_{x}$ and $79 \sigma_{y}$ respectively.

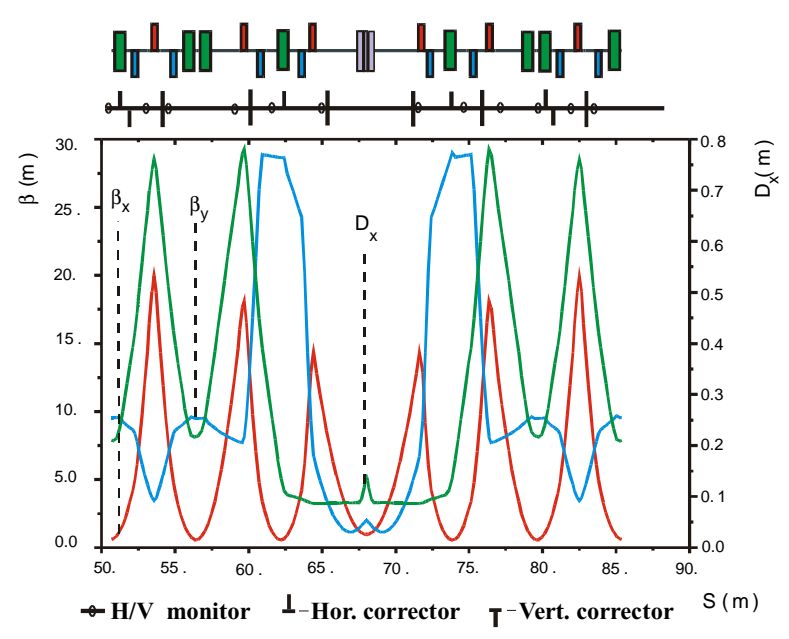

Figure 3: Lattice functions in the wiggler quadrant (wiggler is on).

When the wiggler is on, the dynamic aperture decreases to $64 \sigma_{x}$ and $86 \sigma_{y}$, with the undulator on to $70 \sigma_{x}$ and $78 \sigma_{y}$. In both cases the dynamic aperture is large enough to provide a good lifetime.

\subsection{Closed orbit correction}

For study of the machine with the different sets of errors the MAD computer code was used. All misalignment and field errors were supposed to be Gaussian distributed with cut off at $3 \sigma$. Bending magnets have $1 \mathrm{mrad}$ of misrotation along the longitudinal axis and $5 \cdot 10^{-4}$ error fields; quadrupoles have $200 \mu \mathrm{m}$ of misalignment along the horizontal and vertical axis.

Two schemes for the position of the correctors have been studied. In the first scheme 40 horizontal correctors ( 3 for the matching cell and 2 for the periodic cell) and 32 vertical correctors ( 2 for the matching cell and 2 for the periodic cell) are used. In the second scheme 24 horizontal ( 2 for the matching cell and 1 for the periodic cell) and 32 vertical correctors ( 2 for the matching cell and 2 for the periodic cell) are used. In both schemes 48 horizontal and vertical monitors are used ( 3 for the matching cell and 3 for the periodic cells). Fifty machines have been studied. The maximum closed orbit deviations (CODs) were found $15 \mathrm{~mm}$ and $26 \mathrm{~mm}$ for the horizontal and for the vertical plane respectively. One of the machines was found unstable due the sum coupling resonance.

After the orbit correction was applied (for the lattice with insertion devices off), the maximum horizontal COD was $1.8 \mathrm{~mm}$ for the first scheme and $3 \mathrm{~mm}$ for the second scheme, the vertical closed orbit deviation was $0.99 \mathrm{~mm}$ for the both schemes. The maximum horizontal corrector 
strength is $0.84 \mathrm{mrad}$ in the first scheme and is $0.74 \mathrm{mrad}$ in the second scheme.

The dynamic aperture was calculated for two sets of the errors, one with the maximum COD, and the other with a typical COD. In the first scheme (40 correctors for the horizontal plane and 32 for the vertical one) the dynamic aperture was found $67 \sigma_{x}$ and $91 \sigma_{y}$ for the variant with maximum COD (line plus circles on the Fig.4) and $70 \sigma_{x}$ and $88 \sigma_{y}$ for the variant with the typical COD (line plus squares on the Fig.4). In the second scheme (32 correctors for the horizontal and vertical plane) the dynamic aperture was $58 \sigma_{x}$ and $100 \sigma_{y}, 64 \sigma_{x}$ and $95 \sigma_{y}$ respectively. Thus, the dynamic aperture at the septum is $24 \mathrm{~mm}$, that is enough for effective injection. With the correctors on, the emmitance change is insignificant.

When the wiggler is on, the dynamic aperture is $62 \sigma_{x}$ and $87 \sigma_{y}$ for the error set with the maximum closed orbit deviation (line without symbol on the Fig.4).

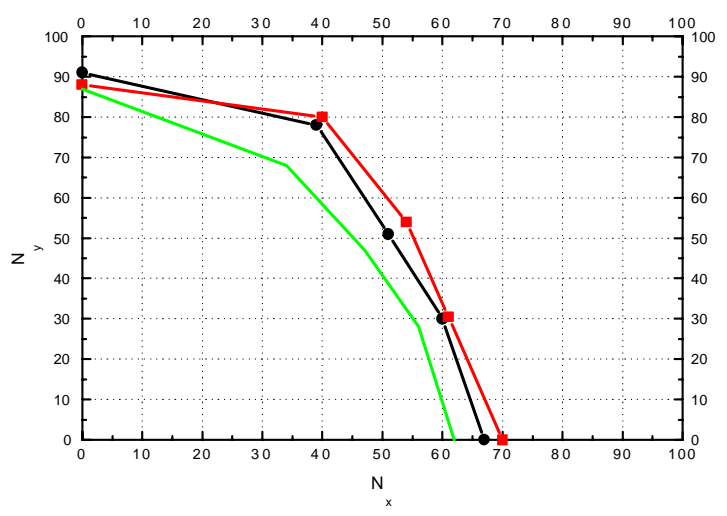

Figure 4: Dynamic aperture expressed in terms of the standard deviations in the presence of the errors.

\section{SYNCHROTRON RADIATION SPECTRA}

The synchrotron radiation from the dipole magnets of DELSY with the maximum brilliance $3.9 \cdot 10^{14}$ photon $/\left(\mathrm{s} \cdot \mathrm{mm}^{2} \cdot \mathrm{mrad}^{2} \cdot 0.1 \%\right.$ b.w.) has rather high intensity in both ultraviolet and infrared regions. This radiation can be used in photoelectron microscopy, time-resolved fluorescent studies of biological objects, in absorption spectroscopy including angular resolved one, in the study of scintillators for nuclear physics and medical applications, in time-resolved VUV luminescence spectroscopy, metrology and photometry. Eight beamlines are planned to be construct for the synchrotron radiation from the bending magnets.

For the beam current of $300 \mathrm{~mA}$ the maximum brilliance from the $10 \mathrm{~T}$ wiggler is $1.6 \cdot 10^{15} \mathrm{photon} /\left(\mathrm{s} \cdot \mathrm{mm}^{2} \cdot \mathrm{mrad}^{2} \cdot 0.1 \% \quad\right.$ b.w.). Hard X-ray radiation from the wiggler with the photon energy 20 $50 \mathrm{keV}$ can be used for the research on VUV luminescence of crystals and pumping of VUV-lasers, time-resolved Moessbauer spectroscopy, EXAFS spectroscopy with soft and hard X-rays, DANES (Diffraction Anomalous Near Edge Structure), DAFS (Diffraction Anomalous Fine Structure), macromolecular crystallography, time-resolved structural studies of biological objects and X-ray microscopy based on multilayer optics. Six beamlines will be constructed for the synchrotron radiation from the wiggler.

The synchrotron radiation from the undulator with maximum brilliance $2.1 \cdot 10^{19}$ photon $/\left(\mathrm{s} \cdot \mathrm{mm}^{2} \cdot \mathrm{mrad}^{2} \cdot 0.1 \%\right.$ b.w.) can be used for VUV luminescence of crystals and pumping of VUV-lasers, for metrology and photometry. For this purpose two beamlines will be constructed.

\section{CONCLUSION}

- Based on the magnetic elements of AmPS DELSY storage ring belongs to the third generation.

- Machine optics is designed in a way to install at least one very strong wiggler with $10 \mathrm{~T}$ magnetic field and one undulator.

- The dynamic aperture is big enough to provide effective injection and good lifetime during the operation with the insertion devices on.

- The scheme of the closed orbit correction allows the correctors from AmPS to be used.

\section{REFERENCES}

[1] I.V.Titkova et al, "Project of the Dubna Electron Synchrotron”, EPAC'2000, Vienna, p.702.

[2] I.V.Titkova et al, "Magnet Lattice of the Synchrotron Radiation Source DELSY", EPAC'2000, Vienna, p.708.

[3] N.A.Mezentsev, private communication.

[4] The MAD Program, Version 8.19, CERN/SL/9013(AP) (Rev.5). 